\title{
Type-I bursts within outbursts of IGR J17473-2721
}

\author{
Y.-P. Chen ${ }^{1}$, S. Zhang ${ }^{1}$, D. F. Torres ${ }^{2}$, J.-M. Wang ${ }^{1,3}$, and T.-P. Li ${ }^{1,4}$ \\ ${ }^{1}$ Laboratory for Particle Astrophysics, Institute of High Energy Physics, Beijing 100049, PR China \\ e-mail: chenyp@mail.ihep.ac.cn \\ 2 ICREA \& Institut de Ciències de l'Espai (IEEC-CSIC), Campus UAB, Facultat de Ciències, Torre C5-parell, 2a planta, \\ 08193 Barcelona, Spain \\ 3 Theoretical Physics Center for Science Facilities (TPCSF), CAS \\ 4 Center for Astrophysics, Tsinghua University, Beijing 100084, PR China
}

Received 6 June 2009 / Accepted 31 October 2009

\section{ABSTRACT}

\begin{abstract}
Aims. Two outbursts were observed by RXTE in the history of the atoll source IGR J17473-2721. During the most recent outburst in 2008, the source showed a complete series of spectral states/transitions. The neutron star system was prolific in type-I X-ray bursts, and we investigate them in the context of complete outbursts evolution.

Methods. A total exposure of $\sim 309$ ks was collected by RXTE during the two outbursts of IGR J17473-2721. We carried out a systematic search for type-I bursts in this data set. For each burst found, we investigated the burst profile, the peak flux, and their dependence on the accretion rate along the evolution of the outbursts.

Results. Eighteen type-I X-ray bursts were found from IGR J17473-2721: two from the outburst in 2005 and the other 16 from the recent outburst in 2008. Among them, 3 bursts show photospheric radius expansion (PRE). The distance to the source is estimated as $6.4 \mathrm{kpc}$ with a 15\% uncertainty based on the three bursts that show PRE. In the recent outburst, there are 6 bursts showing up in the low/hard state prior to the state transition to a high/soft state, 3 bursts at the end phase of the high/soft state, and 7 in the following low/hard state. The blackbody radius of these bursts presents a variety of interesting features. We find that, at the end of the recent outburst, the profile of the blackbody radius is anti-correlated with the blackbody temperature and the burst flux. The durations of the type-I burst are found to correlate with the Eddington ratio and to have two parallel evolution groups. Along the decreasing Eddington ratio, the burst duration decreases and ends in each group the PRE bursts occurred. This provides new clues to the type-I bursts in the context of outbursts for atoll XRBs.
\end{abstract}

Key words. star: neutron - individual: IGR J17473-2721 - X-rays: bursts

\section{Introduction}

Type-I X-ray bursts were discovered during the mid-1970s (Grindlay et al. 1976; Belian et al. 1976), and manifest as a sudden increase (typically a factor of 10 or greater) in the X-ray luminosity of neutron star systems (NSs). Typical type-I X-ray bursts exhibit fast rise times ( $\$ 1$ and $10 \mathrm{~s}$ ), and slower exponential decay (tens to hundreds of seconds). These bursts are caused by unstable burning of accreted $\mathrm{H} / \mathrm{He}$ on the surface of neutron stars in low-mass X-ray binary (LMXB) systems, in contrast to type-II bursts, which are thought to be caused by an increase in the accretion rate from the companion "donor" star. For reviews, see Lewin et al. (1993), Cumming (2004), and Strohmayer \& Bildsten (2006). Recently, Galloway et al. (2008) has presented a large sample of bursts (1187 in total) observed by RXTE over a time interval of more than ten years.

Atoll sources have a lower accretion rate than $\mathrm{Z}$ sources and can have hard states, soft states, and transitions between them. In the hard state, a significant fraction of the energy is emitted above $20 \mathrm{keV}$ and is normally accompanied by radio jets. The source in the soft state has relatively much weaker emission above $20 \mathrm{keV}$ and is believed to be dominated by the accretion disk and the boundary layer where the accreted matter impacts the NS surface. For reviews, see Hasinger \& van der Klis (1989) and van der Klis (2006). Bursts are more prolific in atolls than in $\mathrm{Z}$ sources and have been observed in all of the above X-ray states (e.g., Galloway et al. 2008).
IGR J17473-2721 has been identified as an atoll source (Altamirano et al. 2008b). To date, IGR J17473-2721 has shown two outbursts observed by RXTE, in 2005 and a more recent one in 2008. The 2005 outburst was found to remain in the low/hard state, while the recent one consists of a variety of spectral states/transitions. The source stayed in the low/hard state for 2 months and then transferred to the high/soft state in 3 days; during the decay, the source transferred back to a low/hard state at a lower luminosity level, forming the so-called hysteresis. The spectral analysis may suggest a highly inclined XRB system (Zhang et al. 2009). The long-lived preceding low/hard state makes IGR J17473-2721 resemble the behavior of outbursts seen in black hole X-ray binaries like GX 339-4. For details of RXTE observations on the two outbursts see Zhang et al. (2009). Several type-I bursts were detected in IGR J17473-2721 during the outbursts: 2 type-I X-ray bursts in 2005 were reported by Galloway et al. (2008). Several other bursts detected in the 2008 outburst were first reported by Del Monte et al. (2008), with SuperAGILE, and then by Altamirano et al. (2008a) with SWIFT. Altamirano et al. (2008b) estimate the distance is likely to be between $4.9 \mathrm{kpc}$ (using the theoretical Eddington limit for a pure $\mathrm{He}$ atmosphere) and $5.7 \mathrm{kpc}$ (using the empirical value for the Eddington luminosity from Kuulkers et al. 2003) based on the peak flux of two bursts that show photospheric radius expansion.

Given that IGR J17473-2721 is among the few atoll sources that show a large number of type-I bursts occurring in the context 

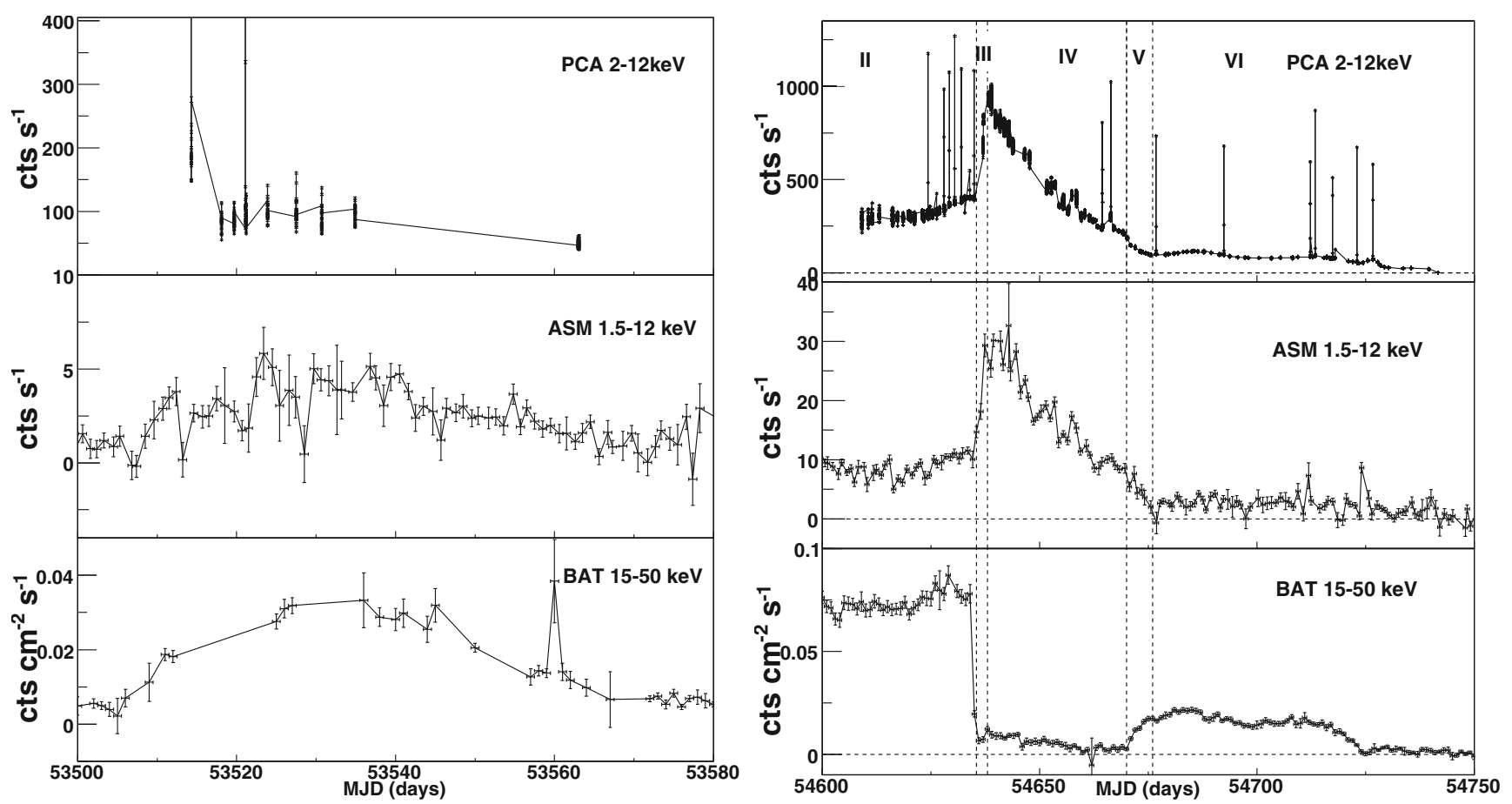

Fig. 1. The PCA(PCU2)/ASM/BAT lightcurves of IGR J17473-2721 during the short outburst (left panel) in 2005 and the recent outburst (right panel) in 2008. The type-I X-ray bursts are the spikes in the PCA lightcurve.

of complete outbursts (Zhang et al. 2009), we carried out the analysis on each type-I burst, based on the most updated RXTE observations and analysis tools. The observation and data analysis are commented on in Sect. 2, the results in Sect. 3, and finally the discussion and summary in Sect. 4.

\section{Observations and data analysis}

Public data from RXTE of IGR J17473-2721 include $130 R X T E /$ PCA pointed observations, with the identifier (OBSID) of proposal number (PN) 91080 and 93442, in the data archive of the High Energy Astrophysics Science Archive Research Center (HEASARC). These observations sum up $\sim 309$ ks of exposure on the source and scatter over the short outburst in 2005 and the long outburst in 2008 (Zhang et al. 2009). The analysis of PCA data was performed by using Heasoft v. 6.2. We filtered the data using the standard $R X T E / P C A$ criteria. To be precise, only the PCU2 (in the 0-4 numbering scheme) was used for the analysis, because only the PCU2 was $100 \%$ during the observation, and we picked up the time intervals under the following constraints on elevation angle $>10^{\circ}$, and pointing offset $<0.02^{\circ}$. Elevation is the angle above the limb of the Earth, and the pointing offset is the angular distance between the pointing and the source. The background file used in the analysis of PCA data is the most recent one available on the HEASARC website for bright sources ${ }^{1}$, and the detector breakdowns have been removed ${ }^{2}$. An additional $1 \%$ systematic error was added to the spectra because of calibration uncertainties, if not otherwise specified. The spectra were fitted with XSPEC v12.3.1 and the model parameters were estimated with $90 \%$ confidence level.

\footnotetext{
1 pca_bkgd_cmbrightvle_eMv20051128.mdl

2 See the website http://heasarc.gsfc.nasa.gov/docs/xte/ recipes/pca_breakdown.html for more information.
}

\section{Results}

We carried out a systematic search for bursts in the lightcurve of IGR J17473-2721. The lightcurve was extracted in $64 \mathrm{~s}$ time bins for every observation identifier (OBSID) with PCA standard 2 model for each energy channel. The lightcurves of the two outbursts are shown in Fig. 1, where the background has been removed. For comparison, we also put together the ASM/RXTE (1.5-12 keV) and BAT/SWIFT (15-50 keV) lightcurves for the two outbursts. The entire 2008 outburst was divided into 6 regions according to the spectral state (following Zhang et al. 2009): regions II/VI for low/hard states, region IV for high/soft state, and regions III/V for the state transitions. The type-I X-ray bursts appear in the PCA lightcurves as spikes, with 2 detected in the 2005 outburst and 16 in the 2008 one. A lightcurve with a time binning of $16 \mathrm{~s}$ shows no additional shorter bursts.

The profile of each type-I X-ray burst was derived in time bins of $1 \mathrm{~s}$ under PCA E mode (E_125us_64M_0_1s, which has $125 \mu$ s time resolution in 64 energy channels starting at channel $0,1 \mathrm{~s}$ read out time) in the $2-60 \mathrm{keV}$ band (Fig. 2). The background and persistent emission were taken off. For each burst, we estimated the peak time and the peak flux from the lightcurves, and the decay time from fitting the decay part of the lightcurve with an exponential shape (Fig. 2). The rise time is defined as the interval during which the flux increases from $10 \%$ to $90 \%$ of the peak value (Galloway et al. 2008). These parameters are presented in Table 1, for each of the bursts. The hardness ratio, defined as the flux ratio $(6-30 \mathrm{keV}) /(2-6 \mathrm{keV})$, is computed for each burst in $0.25 \mathrm{~s}$ time bins (Fig. 3), which is used to investigate the burst evolution (Strohmayer \& Bildsten 2006). Accordingly, 3 bursts $(\# 9,11,18)$ are found to be the PRE events.

The spectra of each burst were extracted with PCA E mode mostly in 3-20 keV for every $0.25 \mathrm{~s}$, based on only PCU 2 data. We took the BBodyrad model to fit the data, with the absorption fixed at $3.8 \times 10^{22}$ atoms $/ \mathrm{cm}^{2}$ (Altamirano et al. 2008a). We used 
Y.-P. Chen et al.: Type-I bursts within outbursts of IGR J17473-2721
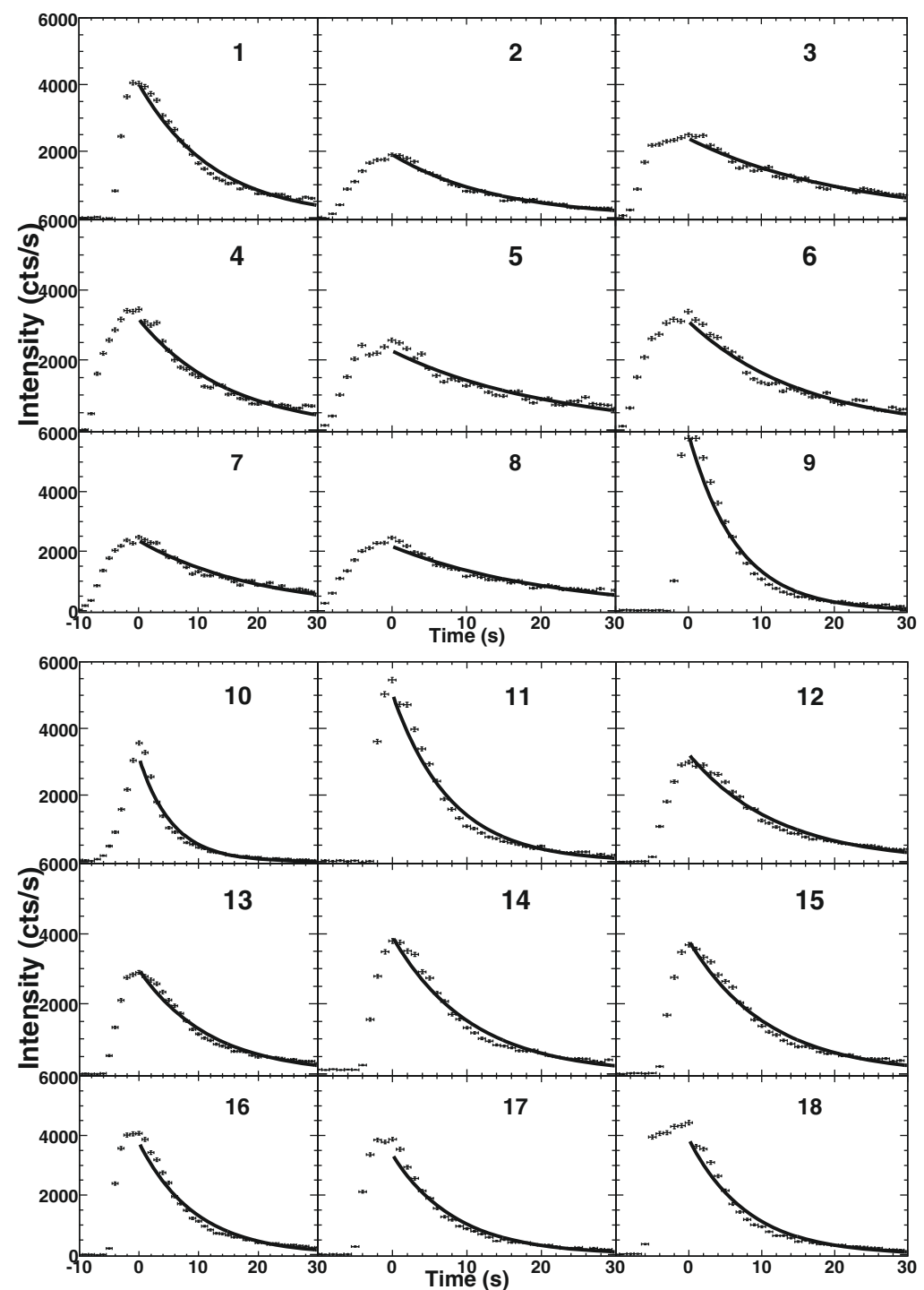

Fig. 2. The profiles and the fit to their decays with an exponential shape for the 18 bursts. The time bin is $1 \mathrm{~s}$.

the spectral data at $100 \mathrm{~s}$ prior to the burst as background, subtracting it from the burst data (as in Muno et al. 2000). The data of the persistent emission are fitted with a model of disk blackbody plus power law, commonly used to model atoll sources, under a fixed column density $3.8 \times 10^{22}$ atoms $/ \mathrm{cm}^{2}$ as derived in Altamirano et al. (2008a) and a fixed iron line at $6.4 \mathrm{keV}$. The reduced $\chi^{2}$ of the fit for each burst are generally $\sim 0.8-1.2$, mostly showing good fits. The resulting evolution of the blackbody temperature $\left(T_{\mathrm{bb}}\right)$ and the corresponding radius $\left(R_{\mathrm{bb}}\right)$ of the emission area are plotted in Fig. 4. The values of $T_{\mathrm{bb}}, R_{\mathrm{bb}}$, and the luminosity $F_{\mathrm{pk}}$ derived at the peak flux, the persistent luminosity $F_{\text {per }}$ prior to each burst, and the bolometric luminosity $E_{\mathrm{b}}$ calculated in the energy band $1.5-30 \mathrm{keV}$ by summing up the luminosity over the entire burst are shown in Table 1. Enclosed as well is the characteristic timescale $\tau$ for each burst, derived as $E_{\mathrm{b}} / F_{\mathrm{pk}}$.

As shown in Fig. 4, the shape of the evolution of $T_{\mathrm{bb}}$ in time always follows the evolution in the burst flux except for the three PRE bursts $(\# 9,11,18)$, where the $R_{\mathrm{bb}}$ curve shows a variety of trends. The $R_{\mathrm{bb}}$ curve monotonically increases along the evolution of the bursts \#1 and 10, showing that the burning expands across the surface of the neutron star. The bursts \#9, 11 , and 18 have an $R_{\mathrm{bb}}$ peak, which is typical of PRE bursts. It is interesting that there are 4 bursts $(\# 14,15,16$, and 17) at the end of the outburst in 2008 that show an anti-correlation between $R_{\mathrm{bb}}$ and the burst flux. The peak temperatures of these bursts are about $3 \mathrm{keV}$, higher than that averaged over the others (about $2.5 \mathrm{keV}$ ). It seems that the burning area in these bursts shrinks with the increasing flux and spreads with the decreasing flux. Actually, such a feature was reported in one burst observed from KS 1731-260 (Muno et al. 2000) and was studied further by Bhattacharyya et al. (2010), using the sample of type-I bursts derived in Galloway et al. (2008). They find an anti-correlation between the burst decaying flux and the blackbody radius for short type-I bursts. The remaining 9 bursts have roughly constant $R_{\mathrm{bb}}$, indicating less change of the burning area across the burst.

To further investigate the burst behavior, we followed the analysis done in Galloway et al. (2008) on the RXTE type-I $\mathrm{X}$-ray bursts. The diagrams of the persistent flux (at $1.5-30 \mathrm{keV}$ ) represented by the Eddington ratio $\gamma=L_{\mathrm{bol}} / L_{E_{\mathrm{dd}}}\left(L_{E_{\mathrm{dd}}}\right.$ takes $3.79 \times 10^{38} \mathrm{erg} \mathrm{s}^{-1}$ from Kuulkers et al. 2003) are shown in Fig. 5, and can be indicative of the accretion rate vs. the time scale $\tau$. We find that $\tau$ is correlated with the Eddington ratio. Along the outburst, the $\gamma-\tau$ evolutions appear in two groups: along the decreasing Eddington ratio, the burst duration decreases and ends up in each group when the PRE bursts occur. 
Table 1. The 18 type-I X-ray bursts' rise time, decay time, the temperature of BB, and the radius, bolometric flux, fluency, characteristic time scale $\tau$, and bolometric flux of the persistent flux.

\begin{tabular}{|c|c|c|c|c|c|c|c|c|c|c|c|}
\hline No & OBSID & $\begin{array}{l}\text { Time } \\
\text { (MJD) }\end{array}$ & $\begin{array}{r}\text { Intensity } \\
\left(\text { cts s }^{-1}\right) \\
\end{array}$ & $\begin{array}{l}t_{r} \\
(\mathrm{~s}) \\
\end{array}$ & $\begin{array}{l}t_{d} \\
(\mathrm{~s}) \\
\end{array}$ & $\begin{array}{c}T_{\mathrm{bb}} \\
(\mathrm{keV})\end{array}$ & $\begin{array}{c}R_{\mathrm{bb}} \\
(\mathrm{km})\end{array}$ & $\begin{array}{c}F_{\mathrm{pk}} \\
\left(10^{-8}\right) \\
\end{array}$ & $\begin{array}{c}E_{\mathrm{b}} \\
\left(10^{-7}\right) \\
\end{array}$ & $\begin{array}{c}\tau \\
(\mathrm{s}) \\
\end{array}$ & $\begin{array}{c}F_{\text {per }} \\
\left(10^{-9}\right) \\
\end{array}$ \\
\hline 1 & $91050-02-01-00$ & 53514.297601 & 4055 & 4 & 12.7 & $2.46_{-0.11}^{+0.11}$ & $7.7_{-0.6}^{+0.7}$ & 6.0 & 12 & 20.0 & 3.30 \\
\hline 2 & $91050-02-05-00$ & 53521.108180 & 1896 & 6 & 13.9 & $2.25_{-0.14}^{+0.15}$ & $6.0_{-0.7}^{+0.8}$ & 2.56 & 5.36 & 20.9 & 2.57 \\
\hline 3 & 93442-01-03-01 & 54624.293851 & 2498 & 6 & 21.7 & $2.33_{-0.13}^{+0.14}$ & $6.6_{-0.7}^{+0.1}$ & 3.61 & 10 & 27.3 & 7.42 \\
\hline 4 & 93442-01-03-05 & 54628.026895 & 3443 & 6 & 15.1 & $2.76_{-0.16}^{+0.17}$ & $5.7_{-0.5}^{+0.6}$ & 5.01 & 10.64 & 27.7 & 8.00 \\
\hline 5 & 93442-01-03-06 & 54629.213273 & 2562 & 5 & 21.5 & $2.54_{-0.16}^{+0.10}$ & $5.7_{-0.6}^{+0.3}$ & 3.74 & 9.12 & 21.2 & 8.02 \\
\hline 6 & 93442-01-04-00 & 54630.523574 & 3378 & 5 & 15.6 & $2.79_{-0.16}^{+0.17}$ & $5.5_{-0.6}^{+0.7}$ & 5.15 & 10.72 & 20.8 & 8.85 \\
\hline 7 & 93442-01-04-01 & 54632.008828 & 2473 & 6 & 20.6 & $2.35_{-0.15}^{+0.16}$ & $6.4_{-0.7}^{+1.0}$ & 3.54 & 9.12 & 25.8 & 9.25 \\
\hline 8 & 93442-01-04-04 & 54634.909141 & 2457 & 7 & 21.4 & $2.53_{-0.18}^{+0.20}$ & $5.6_{-0.9}^{+0.9}$ & 3.51 & 9.12 & 26.0 & 9.21 \\
\hline $9^{*}$ & 93442-01-08-06 & 54664.351270 & 5789 & 2 & 6.7 & $2.32_{-0.09}^{+0.10}$ & $10.1_{-0.7}^{+0.8}$ & 8.23 & 7.36 & 8.9 & 5.42 \\
\hline 10 & 93442-01-08-06 & 54664.486930 & 3563 & 6 & 5.6 & $2.41_{-0.12}^{+0.13}$ & $7.3_{-0.7}^{+0.8}$ & 4.99 & 4.08 & 8.18 & 5.15 \\
\hline $11^{*}$ & 93442-01-09-00 & 54666.359905 & 5058 & 2 & 7.8 & $2.35_{-0.09}^{+0.09}$ & $9.8_{-0.7}^{+0.8}$ & 8.18 & 6.72 & 8.21 & 5.86 \\
\hline 12 & 93442-01-10-05 & 54676.784407 & 2989 & 4 & 12.2 & $2.36_{-0.11}^{+0.12}$ & $7.0_{-0.6}^{+0.7}$ & 4.16 & 7.2 & 17.3 & 2.61 \\
\hline 13 & $93442-01-12-05$ & 54692.425935 & 2898 & 4 & 12.0 & $2.41_{-0.13}^{+0.14}$ & $6.6_{-0.6}^{+0.8}$ & 4.11 & 6.8 & 16.5 & 2.50 \\
\hline 14 & $93442-01-15-04$ & 54712.285321 & 3792 & 3 & 10.6 & $2.76_{-0.15}^{+0.16}$ & $6.0_{-0.5}^{+0.0}$ & 5.85 & 6.08 & 14.4 & 1.79 \\
\hline 15 & $93442-01-15-05$ & 54713.359592 & 3681 & 3 & 10.9 & $2.62_{-0.13}^{+0.13}$ & $6.6_{-0.5}^{+0.6}$ & 5.67 & 7.92 & 14.0 & 2.00 \\
\hline 16 & 93442-01-16-06 & 54717.394303 & 4072 & 3 & 9.5 & $3.25_{-0.17}^{+0.19}$ & $4.8_{-0.4}^{+0.4}$ & 7.01 & 7.92 & 11.3 & 1.70 \\
\hline 17 & 93442-01-17-01 & 54723.019835 & 3875 & 3 & 8.5 & $2.98_{-0.16}^{+0.18}$ & $5.4_{-0.5}^{+0.4}$ & 6.29 & 6.56 & 10.4 & 1.44 \\
\hline $18^{*}$ & 93442-01-17-05 & 54726.704638 & 4430 & 3 & 8.0 & $2.41_{-0.10}^{+0.10}$ & $8.6_{-0.8}^{+0.9}$ & 6.92 & 8.16 & 11.8 & 1.36 \\
\hline
\end{tabular}

Notes. $1 \mathrm{crab}$ is roughly 2500 counts/PCU/s in the $2-60 \mathrm{keV}$ band. The bolometric flux and fluence are presented in the energy band $1.5-30 \mathrm{keV}$, and in unit of erg $\mathrm{cm}^{2} \mathrm{~s}^{-1}$.

${ }^{(*)}$ Burst shows photosphere radius expansion.

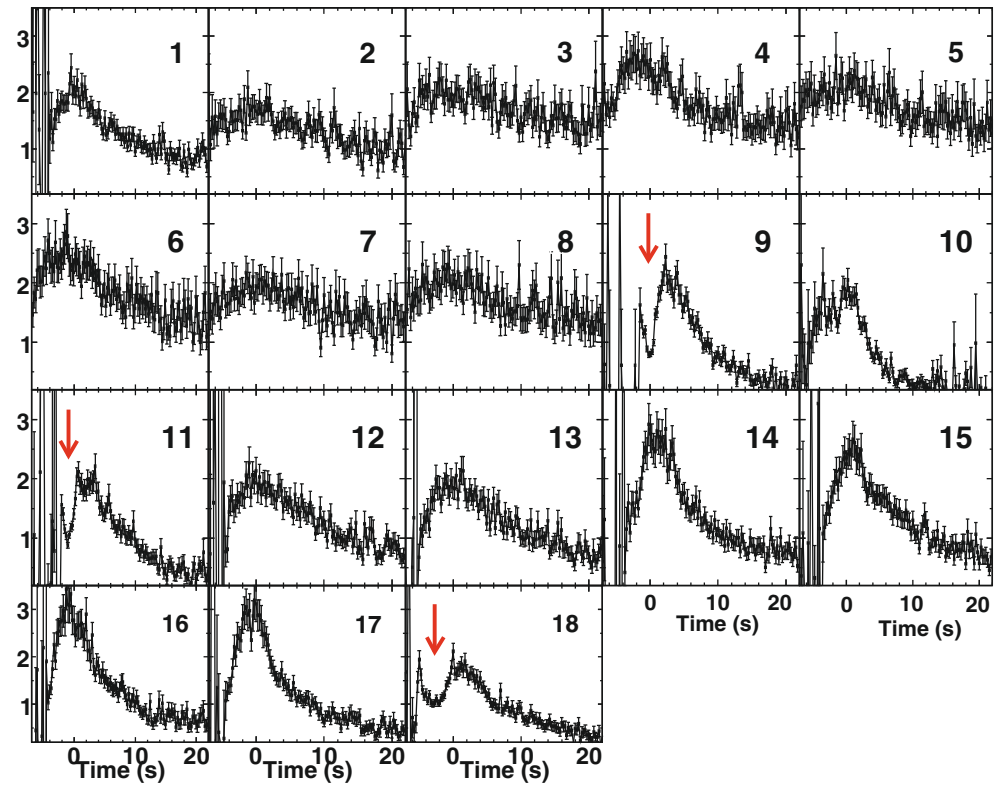

Fig. 3. Hardness ratios $(6-30 \mathrm{keV}) /(2-6 \mathrm{keV})$ of the 18 bursts. The arrows indicate the PRE bursts.

A linear fit to each group shows that the slopes for the two groups are consistent within errors: $419 \pm 19$ for the group at higher $\gamma$ and $469 \pm 131$ for the group with $\gamma$ less than 0.05 .

The accretion rate can be better estimated from the position in the color-color diagram (CCD) (van der Klis 1995) by introducing a parameter so-called $S_{a}$, calculated via the projection to the evolution appears in CCD (Méndez et al. 1999). We calculated PCU2 count rates in four energy bands $(3-4 \mathrm{keV}$, 4-6.4 keV, 6.4-9.7 keV and 9.7-16 keV), with the background subtracted. We define the flux ratio $(4-6.4 \mathrm{keV}) /(3-4 \mathrm{keV})$ as the soft color and the flux ratio $(9.7-16 \mathrm{keV}) /(6.4-9.7 \mathrm{keV})$ as the hard color. In Fig. 6 we show a CCD for the two outbursts of IGR J17473-2721, with each point representing $64 \mathrm{~s}$ of data. The color-color positions of each of the bursts are plotted in Fig. 6. We use the parameter $S_{a}$ to measure the position along the evolution of persistent flux. We set $S_{a}$ to 1 at CCD coordinates $(2.00,0.78)$, and to 2 at $(1.41,0.40)$. For simplicity, we approximate the shape of the color-color diagram with a three-segment broken line. The data close to each burst are projected upon the track, and the derived $S_{a}$ are fitted a Gaussian distribution to estimate the mean $S_{a}$ of each burst. The $S_{a}-\tau$ diagram is then shown in Fig. 7. We find that the $\tau$ is anti-correlated with the $S_{a}$ for the 
Y.-P. Chen et al.: Type-I bursts within outbursts of IGR J17473-2721
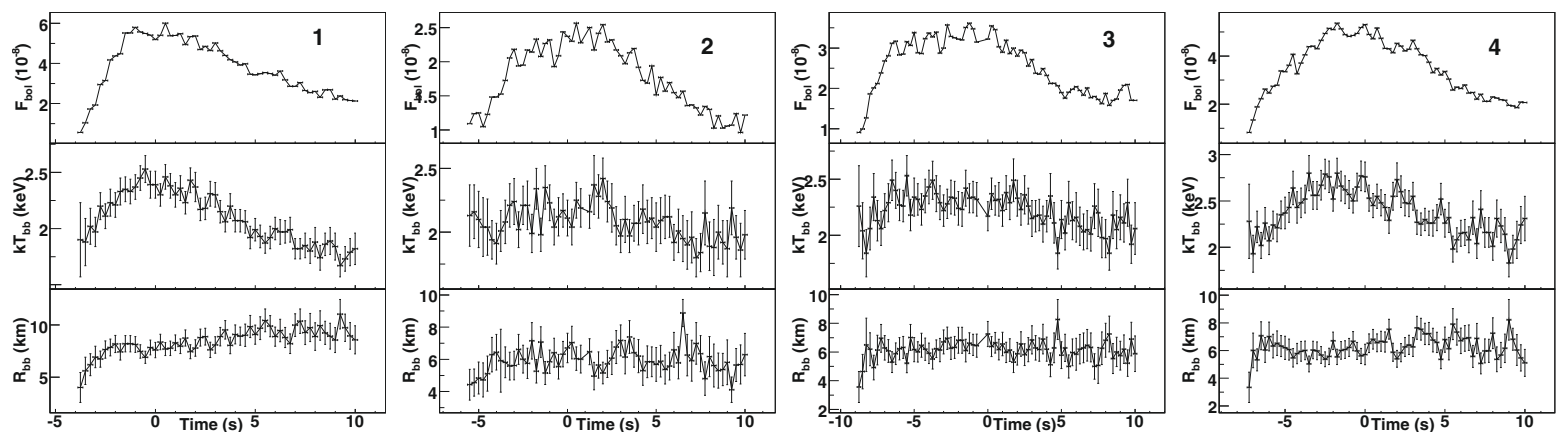

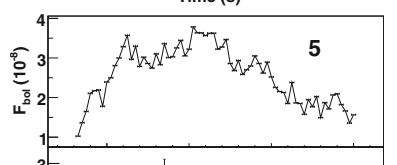
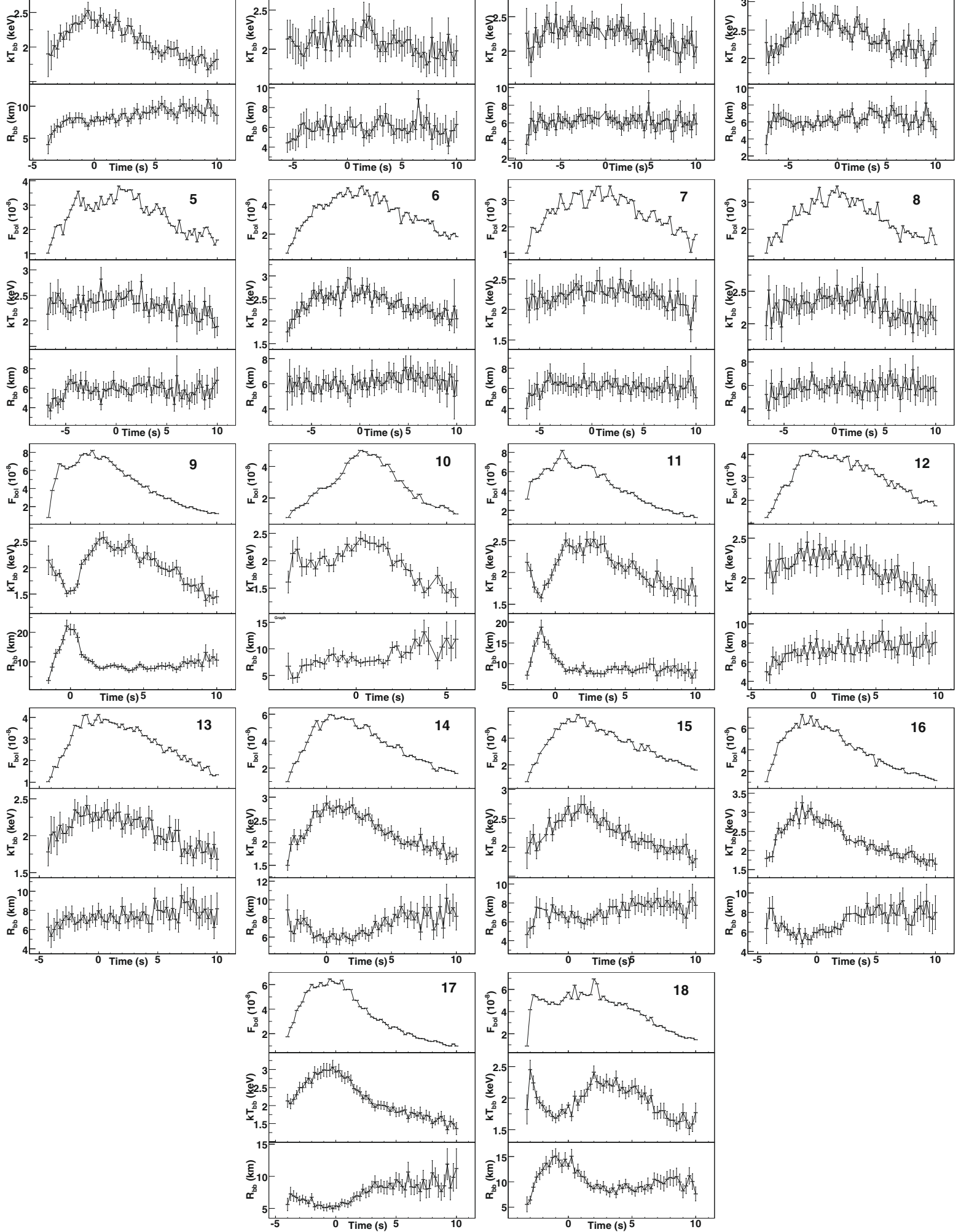

Fig. 4. The evolution of the burst luminosity, temperature, and radius from fitting the data with a blackbody model, for the 18 bursts detected in IGR J17473-2721. The time zero in the X-axis is defined as when intensity reaches the peak (see Table 1). $F_{\text {bol }}$ is the bolometric flux in units of

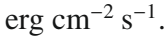

group with large $\gamma$ in the $\gamma$ - $\tau$ diagram (see Fig. 5), indicating an increasing accretion rate. For the group with small $\gamma$, most of $S_{a}$ are around 1.2 and show no obvious evolution in accretion rate except for the last two bursts \#17 and 18. The CCD positions around these two bursts are probably returning to the banana region (see Fig. 6). We investigated these two bursts further via 

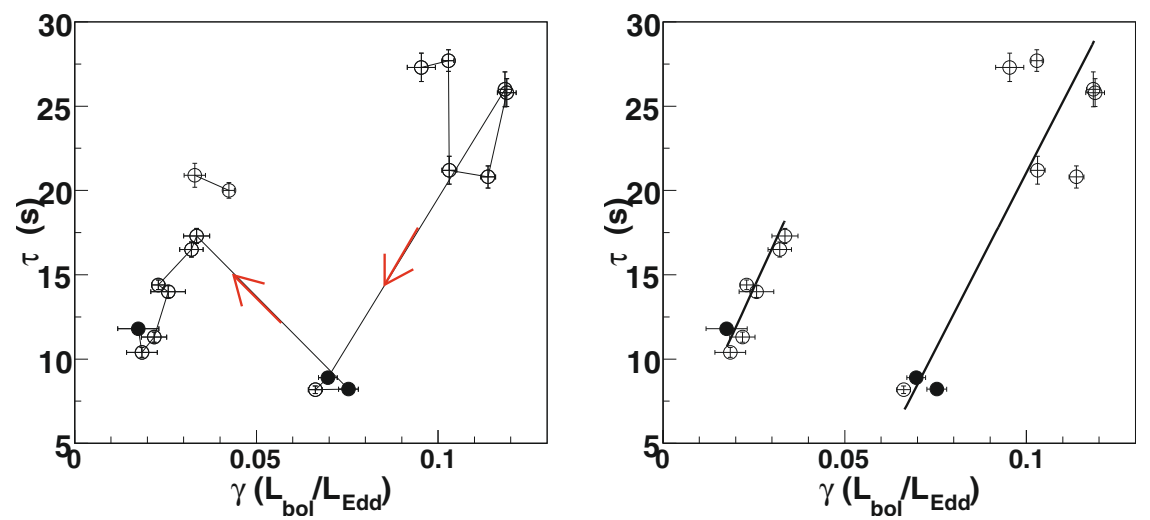

Fig. 5. Characteristic timescale $\tau$ vs. the Eddington ratio $\gamma$. The left panel shows the sequence along the two outbursts, and the right panel shows a linear fit to the two groups of bursts. The filled circles are the three PRE bursts and the open circles the others.

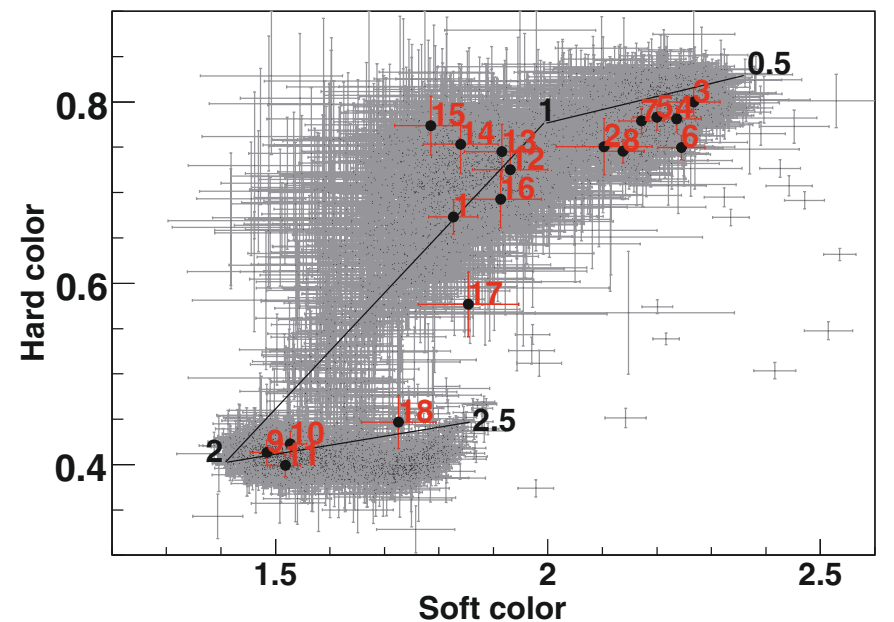

Fig. 6. The color-color diagram of IGR J17473-2721. The integration time is $64 \mathrm{~s}$ per point. The points close to each burst are labeled with a filled circle and a red number. The black solid curve is the atoll-track used to calculate $S_{a}$ (see text), and the locations of $S_{a}=0.5,1,2$ and 2.5 are labeled with black characters.

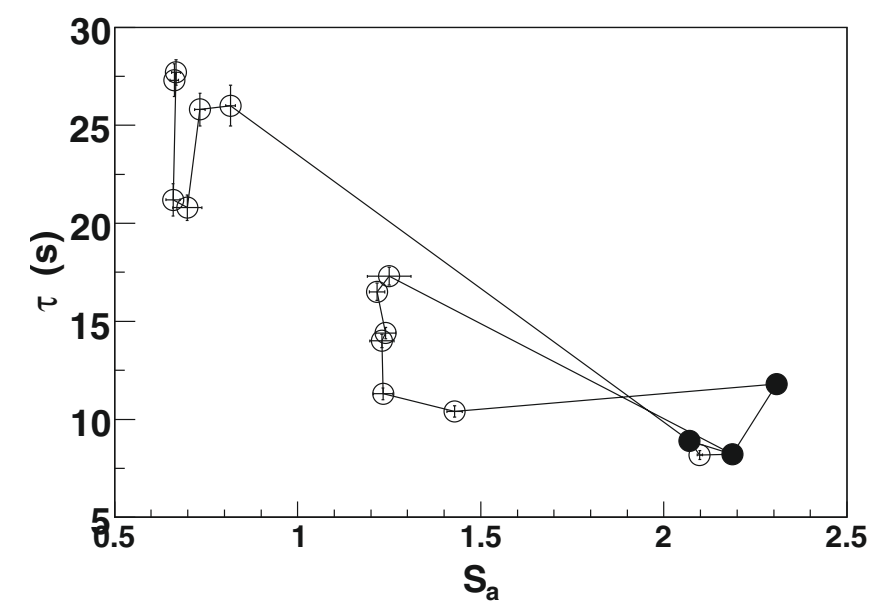

Fig. 7. Characteristic timescale $\tau$ vs. the $S_{a}$. The filled circles are the three PRE bursts and the open circles the others.

timing analysis, and derived the evolutions of the soft and hard color, and the lightcurve in different energy bands (see Figs. 8 and 9). We find that, while the soft color evolves consistently, there is a dip for the hard color within MJD 54720-54730, covering bursts \#17 and 18 (Fig. 8). The lightcurves show that during

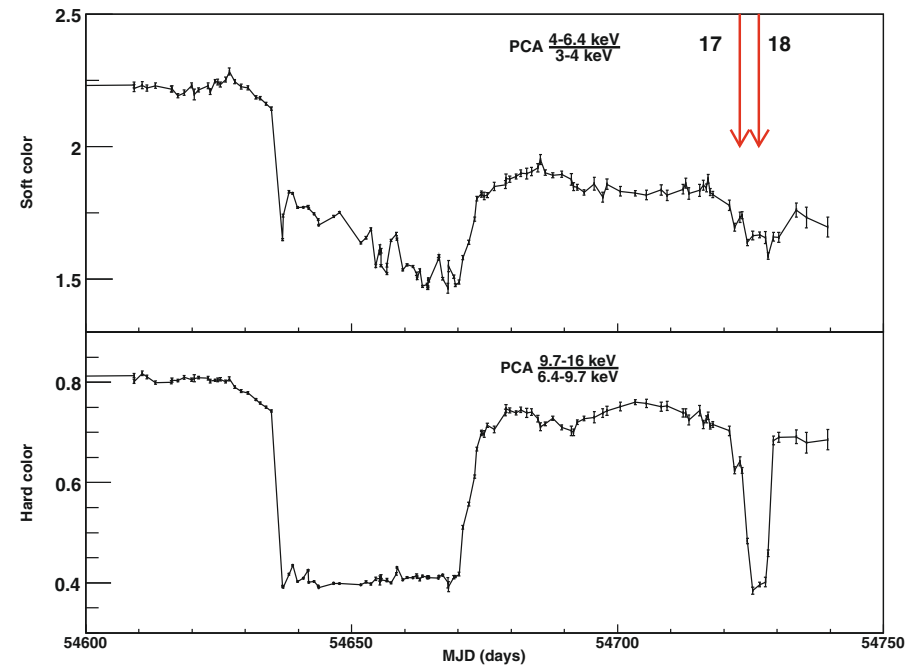

Fig. 8. The evolution of the hardness of the second outburst. The upper panel shows the flux ratio of $(4-6.4 \mathrm{keV}) /(3-4 \mathrm{keV})$, whereas the lower panel shows the flux ratio of $(9.7-16 \mathrm{keV}) /(6.4-9.7 \mathrm{keV})$. The data points are averaged over each OBSID of typical $\sim 3000 \mathrm{~s}$ durations. The locations of burst \#17 and \#18 are marked by arrows.

the decay of the flux at hard X-rays (above $\sim 10 \mathrm{keV}$ ), an additional flare appears at soft X-rays (below $\sim 10 \mathrm{keV}$ ), which leads to a dip in hard color (Fig. 9). We speculate that this additional soft flare might correspond to a flickering of the accretion rate in the disk and that happened on top of the overall decay of the outburst.

\section{Discussion and summary}

During the two outbursts showing up in the observed history of IGR J17473-2721, 18 type-I bursts have been discovered by RXTE in the 3-20 keV band. Apart from the 16 type-I bursts reported by Altamirano et al. (2008b), 7 bursts (\#12-18) are found here at the end phase of the recent outburst. These bursts are investigated in detail within the context of the entire evolution of the outburst, and several features are found.

The rise and decay times of the first 6 bursts (\#3-8) are in general longer than those of the last 7 bursts (\#12-18) in the recent outburst. In both cases the source was in a low/hard state. The shortest time scales of the rise and the decay come from the three bursts $(\# 9,10,11)$ occurred at the end of the high/soft state of the recent outburst. The Eddington ratios in these three time 


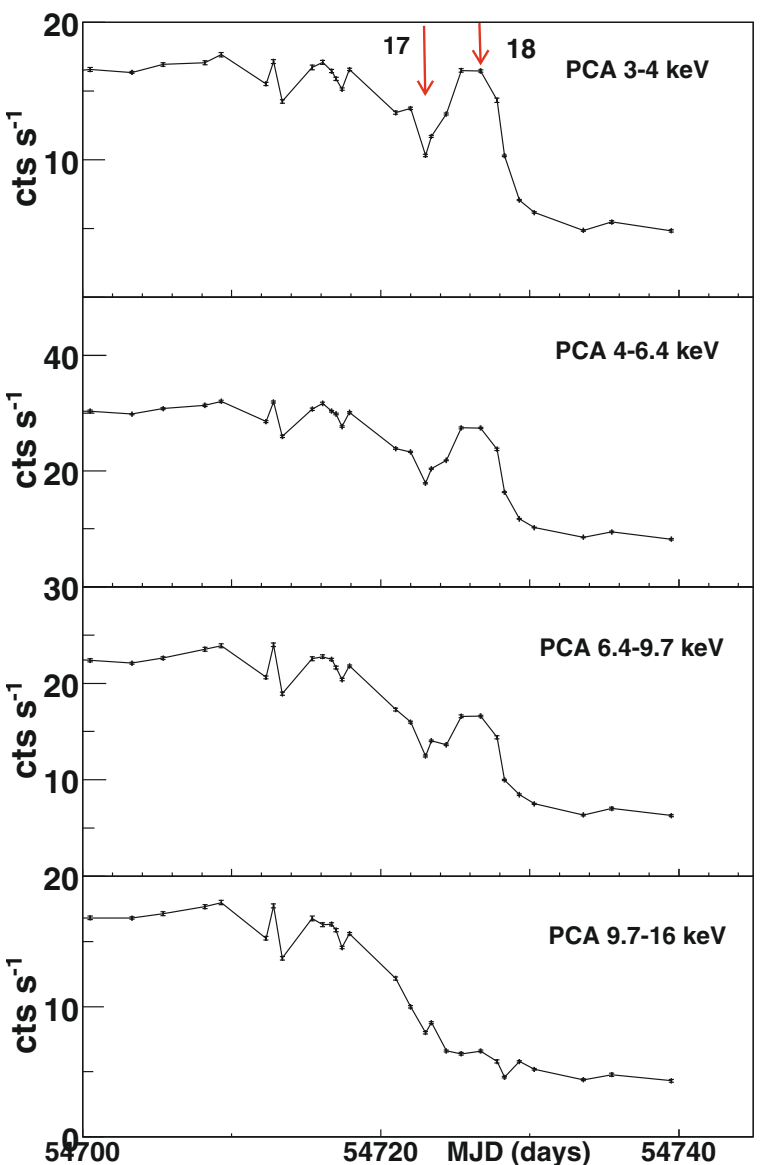

Fig. 9. The flux evolutions of the hard X-ray dip during the decay of the second outburst in four energy bands. The data points are averaged over each OBSID of typical $~ 3000 \mathrm{~s}$ duration. The location of burst \#17 and \#18 are marked by arrows.

regions are about $0.1 L_{E_{\mathrm{dd}}}$ (region II, bursts \#3-8), $0.07 L_{E_{\mathrm{dd}}}$ (region IV, burst \#9-11) and $<0.05 L_{E_{\mathrm{dd}}}$ (region VI, bursts \#12-18). It is obvious that the corresponding burst rate in the low/hard state (region II) prior to the transition to high/soft state is higher than in the remaining part of the outburst (regions III-VI). The net exposure is $\sim 29 \mathrm{ks}$ covering the 6 bursts (\#3-8) in the time interval II (the low/hard state prior to the transition to the high/soft state), and $72 \mathrm{ks}$ enclosing the 7 bursts (\#12-18) in the time interval VI (the low/hard state transferred from the high/soft state). This is consistent with the theoretical prediction for a higher occurrence rate of bursts with increasing accretion rate.

It is thought that burst properties depend on the mass accretion rate and the composition of the accreted matter $(\dot{m})(\mathrm{e} . \mathrm{g}$. Galloway et al. 2008). Since it is given by a He accretor, the burst will always be short irrespective of the accretion rate, which is not the case in IGR J17473-2721. An exception to He accretor is that, for low accretion rates and therefore cool envelopes, He can ignite in a thick layer, and power bursts on timescales of as long as one hour (in't Zand et al. 2009). With a mixed H/He accretor, as might be the case for IGR J17473-2721, the theory predict a correlated accretion rate and burst duration. With a higher accretion rate, the temperature in the fuel layer becomes hotter, leading to an earlier H ignition (Fujimoto et al. 1981). As long as the accretion rate is high enough for hot $\mathrm{CNO}$ cycle burning of the accreted $\mathrm{H}$, since the $\mathrm{CNO}$ cycle is $\beta$ limited, the conversion rate from $\mathrm{H}$ to $\mathrm{He}$ remains the same, so that less $\mathrm{H}$ is consumed before each burst. As the accretion rate goes up, the fraction of $\mathrm{H}$ in the fuel also goes up. This can then account for the increased duration, since it is the $\mathrm{H}$ burning via the rp-process that leads to longer durations. At low enough accretion rates, there will be a rate at which most the accreted $\mathrm{H}$ is processed before ignition. This will lead to He-dominated, shorter bursts. Similar correlation between accretion rate and burst duration has been reported in EXO 0748-676 (Galloway et al. 2008). Here we see that trend for IGR J17473-2721 in the luminosity range $0.01-0.1 L_{E_{\mathrm{dd}}}$, if the Eddington ratio can be the proper indicator of accretion rate: the rise and decay of the bursts are longer at higher values of the Eddington ratios. This is indicated as well in Fig. 5, the plot of the Eddington ratio $\gamma$ vs. the characteristic duration $\tau$, which shows a good correlation.

Along decreasing Eddington ratios, the burst duration becomes shorter, and ends up with the occurrence of the PRE bursts (\#9, 11, and 18), which have the shortest duration. However, we notice that in Fig. 5 such a $\tau-\gamma$ correlation happens twice. After the end of the high $\gamma$ group, the burst jumps to a higher $\tau$ in the low $\gamma$ group, which is hard to understand by considering that the longer burst duration is caused solely by a higher accretion rate. A possible explanation might be that the bursts are mixed $\mathrm{H} / \mathrm{He}$ burns, and the $\mathrm{H} / \mathrm{He}$ fraction can be different in the PRE burst and the subsequent one even if they have the similar Eddington ratio. An alternative choice might be that the PRE bursts might not consume all the He fuel. As shown in Weinberg et al. (2006), the PRE burst is launched at a thin layer at the base of the accreted fuel, since the nuclear energy generation is sensitive to both temperature and density. Above this thin layer is a convective region, where the fuels are consumed. After the PRE burst, both the ashes in the convective region and the fuels outside of this convective region might return to the neutron star surface, contributing to the fuel for the next burst. Also some ashes of the burning are ejected by a radiation-driven wind, and such mass ejection could expose the He-rich material rather than $\mathrm{H}$ (Weinberg et al. 2006). It remains an open question why the PRE bursts occur at the lowest accretion rate at the end of both evolution groups shown in Fig. 5.

The parallel character of the two burst groups in Fig. 5 recalls what shows up in the QPO-luminosity diagram (Méndez et al. 1999), which suggests that the X-ray flux is not always a good indicator of accretion rate. The bursts (\#9, 10, 11, and 18) at the end of both groups are located in the banana region of the $\mathrm{CCD}$, with higher $S_{a}$ values compared to the others that show no clear trend to evolution of accretion rate. For the burst group with higher $\gamma$, the accretion rates within the bursts \#9, 10, 11 are larger than in the earlier ones, but the burst duration goes down. This is, however, inconsistent with the scenario that the accretion rate and the burst duration can be correlated (Fujimoto et al. 1981). A similar case was reported by Muno et al. (2000) on one burst event of KS 1731-260, where the fast burst occurs at a relatively high accretion rate. As pointed out by Muno et al. (2000), such a paradox can be solved by taking the accretion rate per unit area into account at the surface of the neutron star, which is reduced for bursts with a larger burning area (Bildsten 2000).

Actually, we find that, for the burst group with higher $\gamma$ values, the apparent blackbody radius of the bursts \#9, 10, and 11 are larger than those of the earlier ones. Such a trend is also visible in burst \#18 for the burst group with low $\gamma$. The large $R_{\mathrm{bb}}$ means low real local accretion rates, hence low luminosities if the soft X-rays are not dominated by the emission from the accretion disk. Actually, our spectral analysis of outburst from IGR J17473-2721 (Zhang et al. 2009) does show that the soft $\mathrm{X}$-rays are not dominated by the emission from an accretion disk, probably due to the high inclination of the disk. The two 
groups of the bursts, as shown in Figs. 5 and 7, happened in the low/hard state prior to the transition to the high/soft state and in the low/hard state following the high/soft state, respectively. Therefore, they correspond to two different kinds of the low/hard states (the so-called hysteresis) along the outburst of IGR J17473-2721. We notice that the persistent emission around bursts \#17 and 18 have special color evolutions. While the soft color evolves almost continuously, the hard color shows a narrow dip ( $<10$ days) around these two bursts. Such dip is caused by an additional soft flare happen sporadically on top of the decay of the outburst, which may have led to the deviation in CCD or $S_{a}-\tau$ diagram from the overall tracking of the accretion rate for the entire outburst.

PRE bursts are those where the peak flux reach levels comparable to the Eddington luminosity at the surface of the NS. The radiation pressure exceeds the gravitational force binding the outer layers of accreted material to the star. Such bursts frequently exhibit a characteristic spectral evolution in the first few seconds, with a local peak in blackbody radius and a dip in temperature at the same time, while the flux remains approximately constant. This pattern is thought to result from expansion of the X-ray emitting photosphere once the burst flux reaches the Eddington luminosity. The effective temperature must decrease in order to maintain the luminosity at the Eddington limit, and excess burst flux is converted into kinetic and gravitational potential energy in the expanded atmosphere. Consequently, the PRE bursts can be used as distance indicators (Basinska et al. 1984). So far, three such PRE bursts have been detected in IGR J17473-2721, with a peak flux measured within a range of $6.92-8.23 \times 10^{-8} \mathrm{erg} \mathrm{cm}^{2} \mathrm{~s}^{-1}$. We therefore derive a distance of $6.4 \mathrm{kpc}$ with the average of the three peak flux $7.78 \times 10^{-8} \mathrm{erg} \mathrm{cm}^{2} \mathrm{~s}^{-1}$ using the empirically determined Eddington luminosity $3.79 \pm 0.15 \times 10^{38} \mathrm{erg} \mathrm{s}^{-1}$ for PRE burst (Kuulkers et al. (2003) with an uncertainty of 15\%). This is consistent with the previous estimation of the distance with an upper limit of $6.4 \mathrm{kpc}$ by Galloway et al. (2008).

Apart from the three PRE bursts that show the typical evolution of $T_{\mathrm{bb}}$ and $R_{\mathrm{bb}}$ in Fig. 4, the 15 other bursts have the $T_{\mathrm{bb}}$ profiles that can be naturally interpreted as heating resulting from the initial fuel ignition, followed by cooling of the ashes once the available fuel is exhausted. However, the 15 bursts have a variety of evolution trends in $R_{\mathrm{bb}}$, which may indicate that the non-PRE bursts can require more complicated procedure than the PRE ones. Along the burst, the burn can be restricted to an area on the surface of the neutron star, spread steadily along the burst flux evolution, or have an anti-correlation between the burning area and the burst flux. Recently, a very rare triple-peaked burst event was discovered in XRB 4U 1636-53 (Zhang et al. 2009). These authors find that, along the burst evolution, the radius of the burning area increases steadily and shows two dips when the flux got its second and third peaks. These results show that the various trends in radius evolution, which are similar to those found in IGR J17473-2721, can even co-exist within one type-I burst.

We notice that the anti-correlation between the blackbody radius and the decaying flux was seen previously in one burst of KS 1731-260 (Muno et al. 2000). Bhattacharyya et al. (2010) carried out the most recent investigation of such a relation, using a sample of type-I bursts derived in Galloway et al. (2008). They find an anti-correlation between the burst decaying flux and the blackbody radius for short type-I burst, but a positive correlation for a long-duration type-I burst. A possible explanation for this discovery is related to a spectral hardening factor (or the so-called color factor), which accounts for hardening due to the scattering of the photons by the electrons in a neutron star atmosphere. Such a color factor is a function of the chemical composition of the neutron star atmosphere, the actual surface temperature, and the stellar surface gravity. The first two elements could be related to the type-I burst of the different types, $\mathrm{He}$ or $\mathrm{H} / \mathrm{He}$ dominance. Therefore, a change in these may affect the apparent blackbody radius measured in the burst. For IGR J17473-2721, we show at least 4 samples of such a type-I event with anti-correlation between decaying flux and blackbody radius. Their burst durations are shorter than most of the others, which show no trend in correlation between decaying flux and blackbody radius. This is consistent with the discovery in Bhattacharyya et al. (2010) from a sample of 900 bursts from 43 sources. Another possibility is that, since the persistent emission is known to vary, it is possible that some trends seen in inferred radius could be associated with incomplete modeling/subtraction of the persistent emission. A similar issue has been discussed by Muno et al. (2000) in their handling of the background subtraction of the type-I burst of KS 1731-260. There, these authors also adopted the persistent emission $100 \mathrm{~s}$ prior to the burst as the burst background, and ignored the possible variability of the persistent emission during the burst. The possible variability of the persistent emission in each burst, if any, is hard to disentangle from the overall burst emission.

In summary, the 18 type-I X-ray bursts discovered during two complete outbursts of IGR J17473-2721 provide us some new insights into the burst evolution. In particular, the discovery of two similar evolution groups in duration/Eddington ratio diagrams, both ending with the occurrence of the PRE burst at the lowest Eddington ratio, challenges the current modeling of the type-I bursts of XRBs.

Acknowledgements. We are grateful to the anonymous referee for his/her comments, which were helpful in polishing the paper. This work was subsidized by the National Natural Science Foundation of China, the CAS key Project KJCX2-YW-T03, and 973 program 2009CB824800. J.-M. W. thanks the Natural Science Foundation of China for support via NSFC-10325313, 10521001, and 10733010. DFT acknowledges support from grants AYA2009-07391 and SGR2009-811. This research made use of data obtained through the High Energy Astrophysics Science Archive Research Center Online Service, provided by the NASA/Goddard Space Flight Center.

\section{References}

Altamirano, D., Degenaar, N., \& Markwardt, C. 2008a, ATel, 1459 Altamirano, D., Galloway, D., Chenevez, J., et al. 2008b, ATel, 1651 Basinska, E. M., Lewin, W. H. G., Sztajno, M., et al. 1984, ApJ, 281, 337 Belian, R. D., Conner, J. P., \& Evans, W. D. 1976, ApJ, 206, L135 Bhattacharyya, S., Miller, M. C., \& Galloway, D. K. 2010, MNRAS, 401, 2 [arXiv: 0908.4245]

Bildsten, L. 2000, AIPC, 522, 359

Cumming, A. 2004, Nucl. Phys. B Proc. Suppl., 132, 435

Del Monte, E., Evangelista, Y., Feroci, M., et al. 2008, ATel, 1445

Fujimoto, M. Y., Hanawa, T., \& Miyaji, S. 1981, ApJ, 247, 267

Galloway, D. K., Muno, M. P., Hartman, et al. 2008, ApJS, 179, 360

Grindlay, J., Gursky, H., Schnopper, H., et al. 1976, ApJ, 205, L127

Hasinger, G., \& van der Klis, M. 1989, A\&A, 225, 79

in't Zand, J. J. M., Keek, L., Cumming, A., et al. 2009, A\&A, 497, 469

Kuulkers, E., den Hartog, P. R., in't Zand, J. J. M., et al. 2003, A\&A, 399, 663

Lewin, W. H. G., van Paradijs, J., \& Taam, R. E. 1993, Space Sci. Rev., 62, 223

Méndez, M., van der Klis, M., Ford, E. C., et al. 1999, ApJ, 511, L49

Muno, M. P., Fox, D. W., Morgan, E. H., et al. 2000, ApJ, 542, 1016

Strohmayer, T., \& Bildsten, L. 2006, New views of thermonuclear bursts (Compact stellar X-ray sources), 113C156

van der Klis, M. 1995, in X-ray Binaries, ed. W. H. G. Lewin, J. Van Paradijs, \& E. P. J. Van den Heuvel (Cambridge University Press), 252

van der Klis, M. 2006, in Compact Stellar X-ray Sources, ed. W. Lewin, \& M. van der Klis (Cambridge University Press), 39C112

Weinberg, N. N., Bildsten, L., \& Schatz, H. 2006, ApJ, 639, 1018

Zhang, G. B., Mendez, M., Altamirano, D., et al. 2009, MNRAS, 398, 368

Zhang, S., Chen, Y. P., Wang, J. M., et al. 2009, A\&A, 502, 231 